\title{
Functional and Selective RNA Interference in Developing Axons and Growth Cones
}

\author{
Ulrich Hengst, Llewellyn J. Cox, Evan Z. Macosko, and Samie R. Jaffrey \\ Department of Pharmacology, Weill Medical College, Cornell University, New York, New York 10021
}

\begin{abstract}
Developing axons and growth cones contain "local" mRNAs that are translated in response to various extracellular signaling molecules and have roles in several processes during axonal development, including axonal pathfinding, orientation of axons in chemotactic gradients, and in the regulation of neurotransmitter release. The molecular mechanisms that regulate mRNA translation within axons and growth cones are unknown. Here we show that proteins involved in RNA interference (RNAi), including argonaute-3 and argonaute-4, Dicer, and the fragile $\mathrm{X}$ mental retardation protein, are found in developing axons and growth cones. These proteins assemble into functional RNA-induced silencing complexes as transfection of small interfering RNAs selectively into distal axons results in distal axon-specific mRNA knock-down, without reducing transcript levels in proximal axons or associated diffusion of small interfering RNA into proximal axons or cell bodies. RhoA mRNA is localized to axons and growth cones, and intra-axonal translation of RhoA is required for growth cone collapse elicited by Semaphorin $3 \mathrm{~A}$ (Sema3A), an axonal guidance cue. Selective knock-down of axonal RhoA mRNA abolishes Sema3A-dependent growth cone collapse. Our results demonstrate functional and potent RNAi in axons and identify an approach to spatially regulate mRNA transcripts at a subcellular level in neurons.
\end{abstract}

Key words: RhoA; RNA interference; small interfering RNA; Dicer; Fragile X mental retardation protein; axons; growth cones

\section{Introduction}

Protein synthesis occurs in the cell body as well as in axons and growth cones during neuronal development. Axons and growth cones contain polyadenylated mRNAs and ribosomes (Kleiman et al., 1994; Olink-Coux and Hollenbeck, 1996; Steward, 2002), and isolated axons are capable of translating endogenous (Eng et al., 1999) and heterologous (Brittis et al., 2002) mRNAs into proteins. mRNAs encoding proteins such as $\beta$-actin and RhoA have been identified in developing axons (Olink-Coux and Hollenbeck, 1996; Bassell et al., 1998; Wu et al., 2005). Additionally, axonally synthesized proteins are capable of being exported and inserted into the plasma membrane (Brittis et al., 2002), and axons may contain unconventionally organized Golgi bodies (Yamada et al., 1971).

This intra-axonal, or "local," mRNA translation is required for the response of growth cones to guidance cues, such as netrin-1 and Sema3A (Campbell and Holt, 2001; Wu et al., 2005). Recently, axonally localized RhoA transcripts were identified as the target of Sema3A signaling in growth cones, and local translation of RhoA mRNA was shown to be required for Sema3Amediated growth cone collapse (Wu et al., 2005). The requirement for local mRNA translation is also evident in attractive turning elicited by netrin-1, another guidance cue, in which ribosomal inhibitors block the ability of axons to turn and orient

\footnotetext{
Received Dec. 7, 2005; revised March 30, 2006; accepted April 19, 2006.

This work was supported by National Institutes of Health Grant MH066204, the FRAXA Foundation, the Christopher Reeve Paralysis Foundation (S.R.J.), and the Medical Scientist Training Program (E.Z.M.).

Correspondence should be addressed to Samie R. Jaffrey at the above address. E-mail: srj2003@med.cornell.edu. DOI:10.1523/JNEUROSCI.5229-05.2006

Copyright $\odot 2006$ Society for Neuroscience $\quad$ 0270-6474/06/265727-06\$15.00/0
}

within gradients of netrin-1 (Campbell and Holt, 2001; Ming et al., 2002). In another study, protein synthesis was stimulated in axons crossing the spinal midline, as measured using a reporter containing the EphA2 3'UTR (Brittis et al., 2002). Thus, local translation is crucially involved in key processes in axonal development.

The molecular mechanisms that regulate mRNA translation in axons are unknown. A pathway that regulates mRNA translation is the RNAi pathway (for review, see Mello and Conte, 2004). RNAi uses $\sim 21$ nucleotide RNAs that either repress the translation or trigger the degradation of specific target mRNAs (Mello and Conte, 2004). Dicer, a ribonuclease specific for doublestranded RNA, cleaves long double-stranded RNAs into small interfering RNAs (siRNAs) and endogenously transcribed stemloop RNAs into microRNAs (Mello and Conte, 2004). Individual strands of Dicer-generated RNA duplexes, termed guide strands, are incorporated into RNA-induced silencing complexes (RISCs). RISCs can result in target mRNA cleavage if the guide strand exhibits a high degree of complementarity with target $\mathrm{mR}$ NAs, or translational repression if the guide RNA exhibits partial complementarity (Mello and Conte, 2004). Defects in Dicer result in abnormal brain development and axon guidance abnormalities in zebrafish (Giraldez et al., 2005), suggesting a role for siRNAs or microRNAs in neuronal development. Indeed, numerous brain-specific miRNAs have been identified that exhibit spatial and temporal expression patterns that correlate with major neurodevelopmental stages (Krichevsky et al., 2003).

To address the role of miRNAs in axonal development, we explored the localization of proteins involved in mediating RNAi in growth cones. Additionally, we examined the ability of developing axons to perform siRNA-mediated mRNA knock-down. 
Our findings indicate that developing axons exhibit functional RNAi, and suggest an important role for microRNAs in the regulation of axonal mRNA translation.

\section{Materials and Methods}

Cell cultures. Reagents were from Sigma (St. Louis, MO), unless otherwise indicated. Embryonic day 15 (E15) rat DRG cultures were prepared as described previously (Wu et al., 2005). For immunofluorescence experiments, DRG explants or dissociated cells were plated on poly-Dlysine (PDL)/laminin-coated glass coverslips (BD Biosciences, Franklin Lakes, NJ). After $3 \mathrm{~d}$ in vitro (DIV), cultures were fixed with $4 \%$ paraformaldehyde in TBS (20 mm Tris, $\mathrm{pH} 8.0,150 \mathrm{~mm} \mathrm{NaCl}$ ) for $30 \mathrm{~min}$ at $25^{\circ} \mathrm{C}$, permeabilized with $0.5 \%$ Triton $\mathrm{X}-100 / \mathrm{TBS}$ for $10 \mathrm{~min}$, and blocked in 2\% BSA/TBS for $30 \mathrm{~min}$. Immunofluorescence (IF) and immunoblotting [Western blot (WB)] were performed using antiargonaute-3 (Ago 3), anti-Ago 4 [both 1:200 (IF), 1:1000 (WB); Abcam, Cambridge, UK), anti-Dicer [1:50 (IF), 1:100 (WB), unconcentrated tissue culture supernatant clone 13D6; Genetex, San Antonio, TX], or antifragile X mental retardation protein (FMRP) [1:1000 (IF), 1:2000 (WB); Chemicon, Temecula, CA]. Secondary antibodies were AlexaFluor 488 and 568 (Invitrogen, Eugene, OR). All images were obtained using a Nikon (Tokyo, Japan) TE2000 microscope, using MetaMorph (Universal Imaging, Downingtown, PA) for image acquisition with only linear adjustments.

siRNA transfection in axons. Campenot chambers were prepared as described previously (Campenot, 1977), with modifications as follows. DRG explants were plated in the central compartment of three chamber Campenot chambers (Tyler Research, Edmonton, Alberta, Canada) plated on Permanox slides (Lab-Tek; NUNC, Roskilde, Denmark) precoated with collagen, PDL, and laminin. Axons arrived into the side compartment by $5 \mathrm{DIV}$, at which point they were transfected with previously described RhoA-specific or a nontargeting siRNA (Wu et al., 2005), using GeneSilencer (Genlantis, San Diego, CA) transfection reagent in a volume of $50 \mu \mathrm{l}$, according to the manufacturer's protocol. The incorporation of siRNAs into DRG axons was verified by fluorescence microscopy in neurons transfected with a nontargeting FITC-labeled siRNA. Eight hours before fixation, Alexa 488-wheat germ agglutinin (WGA) (Invitrogen) was added to the side chambers to label the axons. Immunofluorescence and quantification of RhoA and fluorescent in situ hybridization (FISH) for RhoA transcripts were performed as described previously (Wu et al., 2005) $48 \mathrm{~h}$ after siRNA transfection. Experiments performed in the absence of WGA revealed identical results, ruling out WGA-dependent alteration in mRNA stability or RNAi (E. Z. Macosko and S. R. Jaffrey, unpublished data).

For collapse assays, phase-contrast pictures of growth cones were taken before and after incubation with Sema3A ( $450 \mathrm{ng} / \mathrm{ml})$ or vehicle in the side chamber for $1 \mathrm{~h}$. Analysis of the collapse data was done as described previously (Wu et al., 2005).

\section{Results \\ Proteins implicated in RNAi are localized to DRG axons and growth cones}

To determine whether RNA interference could have a role in local translation in axons, we examined whether proteins involved in RNAi are found in developing axons. A core component of RISC is the argonaute proteins, which contain PAZ domains that bind guide RNAs (for review, see Tanaka Hall, 2005). We examined the localization of endogenous argonaute proteins in axons by immunofluorescence using Ago 3 and Ago 4-specific antibodies (supplemental Fig. $1 \mathrm{~A}$, available at www.jneurosci.org as supplemental material). Rat DRG dissociated neurons were prepared from E15 embryos, the time at which DRG axons elongate and begin pathfinding. Argonaute- 3 and argonaute- 4 were present most prominently in cell bodies but were clearly visible in axons and growth cones, in which they displayed a discrete punctate localization (Fig. 1A,B,E-J; supplemental Fig. 2, available at www.jneurosci.org as supplemental material). The punctate dis-
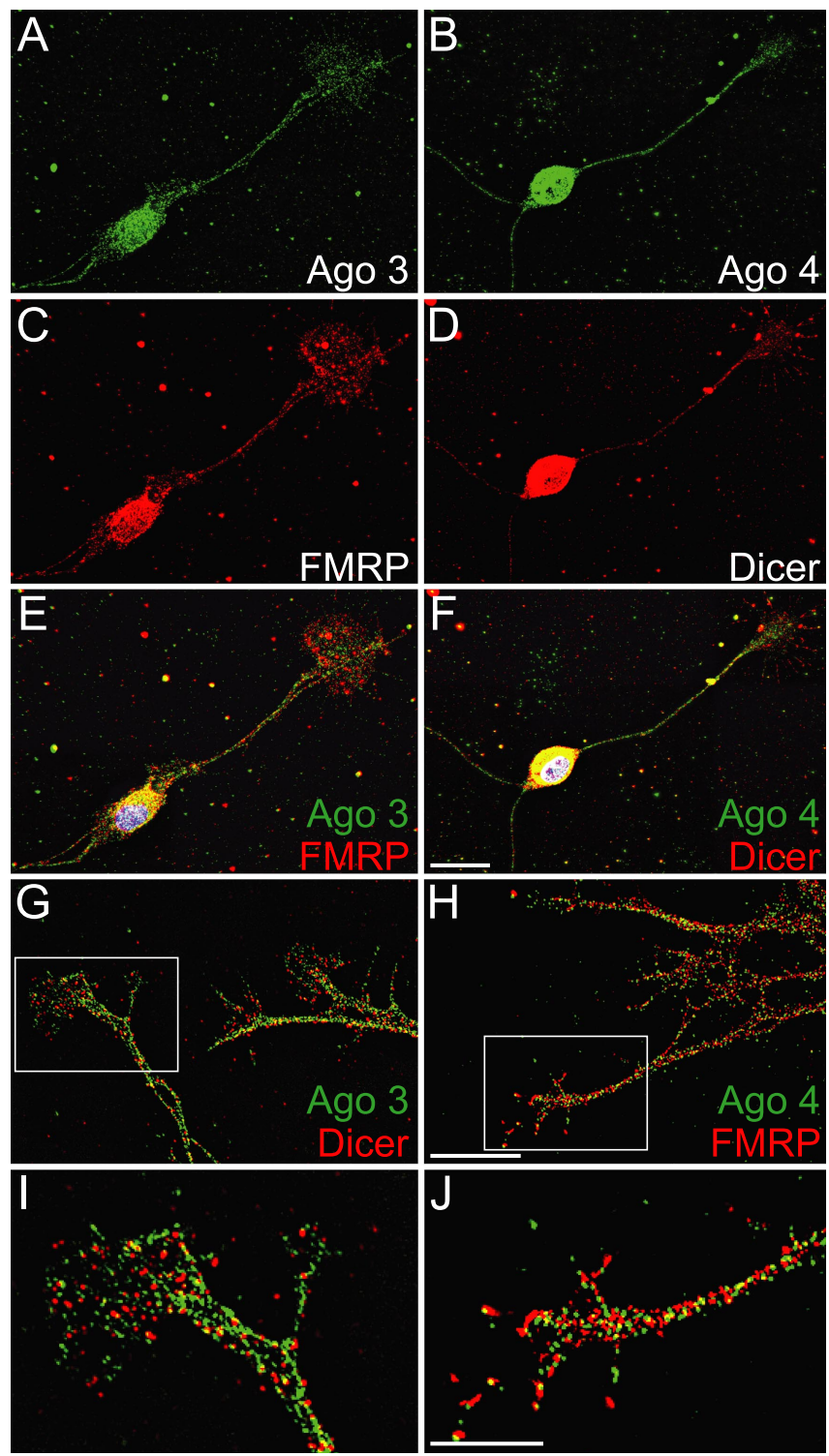

Figure 1. Localization of RNAi-related proteins in axons and growth cones. A-D, E15 dissociated rat DRG neuronal cultures (DIV 3 ) were labeled with antibodies directed against argonaute $3(\boldsymbol{A})$, argonaute $4(\boldsymbol{B}), \operatorname{FMRP}(\boldsymbol{C}$ ), and Dicer (D). $\boldsymbol{E}$, Colocalization of Ago 3 (green), FMRP (red), with 4', $6^{\prime}$-diamidino-2-phenylindole dihydrochloride (DAPI; blue). $\boldsymbol{F}$, Colocalization of Ago 4 (green), Dicer (red), with DAPI (blue). Scale bar, $10 \mu \mathrm{m}$. $\boldsymbol{G}, \boldsymbol{H}$, Each of these proteins exhibit punctate localization in both axons and growth cones. Scale bar, $10 \mu \mathrm{m}$. I, J, Highpower magnification of the growth cones boxed in $\boldsymbol{G}$ and $\boldsymbol{H}$. Scale bar, $5 \mu \mathrm{m}$.

tribution of these proteins is reminiscent of the localization of axonal mRNAs, Staufen, and ribosomal proteins, which are found in discrete, mobile RNA-containing granular structures, termed "RNA granules" (Kanai et al., 2004). These structures were previously characterized in dendrites and appear to have roles in RNA translation or storage (Krichevsky and Kosik, 2001).

We also examined the localization of FMRP in axons. FMRP is a repressor of mRNA translation that is absent in the fragile $\mathrm{X}$ mental retardation syndrome and has been found to be associated with several proteins implicated in RNAi (Caudy et al., 2002; Ishizuka et al., 2002; Jin et al., 2004b), although the specific role of FMRP in RISC is not yet known. FMRP has previously been found in dendrites (Weiler et al., 1997), but its localization in developing axons has not been addressed. As with argonaute-3 and argonaute-4, FMRP was found in axons in a punctate distri- 
bution (Fig. 1C,E,H). The punctate distribution of FMRP in developing axons is similar to the distribution of FMRP in dendrites (Antar et al., 2005).

The finding that argonautes are present in developing axons indicates a potential role for RISC in the regulation of axonal mRNA translation. For RISC to be functional, Dicer is required for dsRNA processing and generation of guide RNAs. These guide RNAs could be exclusively generated in the soma and then transported to axons, or they could be synthesized locally. To determine whether dsRNAs could be processed in axons, we examined the localization of endogenous Dicer with an antibody that recognizes Dicer, as well as proteins that may reflect its alternatively spliced gene products (Irvin-Wilson and Chaudhuri, 2005) (supplemental Fig. 1C, available at www.jneurosci.org as supplemental material). Dicer was detected in axons and growth cones in a highly specific punctate localization that, like FMRP, did not overlap with argonautes (Fig. $1 D, F, G, I$ ).

\section{Assembly of functional RISC in developing axons}

The localization of these RNAi-associated proteins in axons suggests that RNAi has a role in developing axons. To determine whether RNAi is functional in axons, we used compartmentalized Campenot chambers (Campenot, 1977) to segregate axons from their cell bodies (Fig. 2A). E15 DRG explant cultures were plated in the central compartment of Campenot chambers. A gradient of NGF was established to promote the growth of axons into the side compartment through a thinly applied silicone grease layer that seals the chamber with the plastic slide. To create this gradient, DRGs were plated such that the concentration of NGF in the central compartment was $10 \mathrm{ng} / \mathrm{ml}$, and the concentration in the side compartment was $100 \mathrm{ng} / \mathrm{ml}$. Axons cross into the side compartment by $5 \mathrm{DIV}$, at which point NGF is eliminated from the central compartment to induce death of neurons that have not crossed. Immunofluorescence analysis with antibodies directed against RISC-associated proteins demonstrated their presence in axons and growth cones at this time point (supplemental Fig. 3, available at www.jneurosci.org as supplemental material). To label axons that have crossed in the side compartment, we added Alexa 488-conjugated WGA, a retrograde tracer, to the side compartment. This results in labeling of distal axons, which reside in the side compartment, as well as retrograde labeling of contiguous proximal axons and cell bodies in the central compartment.

To determine whether RNAi can occur directly within axons, we assessed whether transfection of RhoA mRNA-specific (Wu et al., 2005) siRNA exclusively into the side compartment could specifically knock down axonal RhoA protein and RhoA mRNA. Transfection in the side chamber of an siRNA directed against RhoA mRNA, but not of a control siRNA, leads to a reduction of axonal RhoA protein to background levels (Fig. $2 B-E$ ). Transfection of RhoA-specific siRNA in the side compartment resulted in a $>75 \%$ reduction in RhoA mRNA levels in distal axons (Fig. $2 J, K, R$ ) as assessed by FISH, whereas RhoA mRNA levels in distal axons were not significantly altered by transfection of a nontargeting siRNA (Fig. $2 H, I, R$ ). The absence of RhoA mRNA knockdown by transfection of the control siRNA indicates that siRNA transfection does not induce a nonspecific mRNA degradation or retrograde trafficking response. The knock-down of RhoA mRNA levels was also specific to the side compartment, because transfection of RhoA siRNA into the side compartment did not induce knock-down of RhoA mRNA in contiguous axons in the central compartment but was associated with an increase in transcript levels (Fig. $2 L-O, R$ ). This may reflect a compensatory up- regulation of RhoA transcription in cell bodies in response to decreased RhoA activity in axon terminals.

The finding that compartmentalized transfection leads to distal axon-specific mRNA knock-down suggests that siRNA that is transfected into axons functions locally and is not transported into proximal axons or cell bodies. To further examine the basis for the spatially restricted action of siRNA, we transfected FITClabeled control siRNA into the side chamber and examined the lateral diffusion of these siRNA after $48 \mathrm{~h}$. In these experiments, FITC-labeled siRNA appeared as fluorescent puncta throughout the distal axons, with minimal transport into proximal axons (Fig. 2P,Q). Indeed, fluorescent siRNA does not traverse the 1 $\mathrm{mm}$ width of the partition and is only detected up to $20 \mu \mathrm{m}$ beyond the edge of the partition facing the side compartment. Thus, siRNA appears to be incorporated into relative immobile punctate structures within axons, and does not diffuse throughout the axons, which may account for the observed spatial specificity of RNAi in axons. siRNA that is not incorporated into ribonucleoprotein structures may be too unstable to be retrogradely trafficked over the time period of these experiments. Together, the ability of axonally transfected RhoA-specific siRNA to specifically induce knock-down of RhoA protein and mRNA indicates that functional RISC complexes can form, be recruited to RhoA transcripts, and elicit RhoA mRNA degradation in axons.

\section{Axon-specific knock-down of RhoA mRNA blocks Sema3A- mediated growth cone collapse}

Axonal RhoA mRNA transcripts have been implicated in Sema3A-mediated growth cone collapse (Wu et al., 2005). In this study, the role of axonally localized RhoA transcripts was addressed by using siRNA to knock down entire neuronal RhoA levels, and then by infecting these neurons with Sindbis viral constructs that expressed heterologous RhoA transcripts that lacked axonal targeting elements. In these neurons, RhoA mRNA was limited to the cell body, and Sema3A exhibited markedly diminished growth cone collapsing activity (Wu et al., 2005). This approach required generation of RhoA transcripts that were both incapable of being targeted to axons and which were resistant to the siRNA used to knock down endogenous RhoA mRNA. Conceivably, compartmentalized siRNA transfection to obtain axon-specific RhoA knock-down could allow a more straightforward assessment of the role of RhoA in growth cone collapse. Thus, we transfected RhoA siRNA into one side compartment and a control, nontargeting siRNA into the other side compartment and monitored Sema3A-dependent growth cone collapse $48 \mathrm{~h}$ later. In axons transfected with RhoA siRNA, baseline collapse rates were significantly reduced, and Sema3A-dependent collapse was markedly diminished, whereas in axons transfected with control siRNA, Sema3A exhibited normal levels of growth cone collapse (Fig. 2S). These studies indicate that axonal RhoA mRNA is required for the ability of Sema3A to induce growth cone collapse.

\section{Discussion}

Intra-axonal mRNA translation has roles in numerous aspects of axonal development, but mechanisms by which extracellular signals regulate mRNA translation in axons are unknown. In this study we find that key proteins involved in the RNAi pathway are found in developing axons. Furthermore, we find that transfection of exogenous siRNA selectively into distal axons results in RNA silencing, suggesting that RISC complexes can assemble and function in axons. In addition to RhoA mRNA, other axonal transcripts can also be knocked down using compartmentalized 



$S$

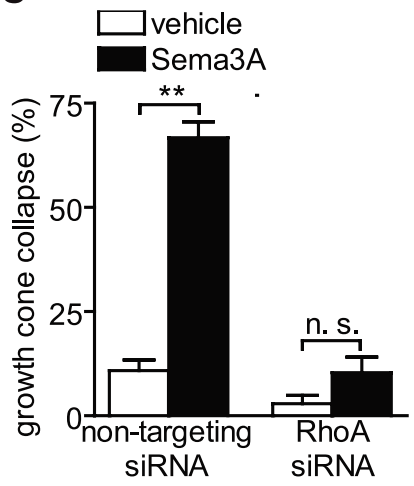

Figure 2. Selective knock-down of mRNA in axons. $A$, Schematic diagram of compartmentalized siRNA transfection in Campenot culturing chambers. Dissociated E15 DRG neurons were plated in the central compartment of Campenot chambers, in the presence of $10 \mathrm{ng} / \mathrm{ml} \mathrm{NGF}$. Axon growth into the side compartments is promoted by inclusion of NGF at $100 \mathrm{ng} / \mathrm{ml}$ in the side chambers. Axons cross by DIV 5 , at which point NGF is removed from the central compartment to induce the death of neurons whose axons have not yet crossed. $\boldsymbol{B}-\boldsymbol{E}$, RhoA immunofluorescence staining reveals knock-down of axonal RhoA protein caused by transfection of RhoA-specific siRNA into the side chamber (D) but not by a control siRNA $(\boldsymbol{B})$. The axons were visualized by GAP-43 immunofluorescence $(\boldsymbol{C}, \boldsymbol{E})$. $\boldsymbol{F}-\mathbf{O}, \mathrm{FlSH}$ with a scrambled $(\boldsymbol{F}, \boldsymbol{G})$ or a RhoA mRNA-specific probe $(\boldsymbol{H}, \boldsymbol{J}, \boldsymbol{L}, \boldsymbol{N})$ of axons in the side $(\boldsymbol{H}-\boldsymbol{K})$ or the central compartment $(\boldsymbol{L}-\mathbf{O})$. The axons are visualized by labeling with Alexa 488 -WGA $(\boldsymbol{G}, \boldsymbol{I}, \boldsymbol{K}, \boldsymbol{M}, \mathbf{O})$. Transfection of RhoA-specific siRNA in the side compartment results in RhoA mRNA knock-down in the axons that have crossed into the side compartment $(\boldsymbol{J}, \boldsymbol{K})$ but not in contiguous axons in the central compartment $(\boldsymbol{N}, \mathbf{O})$. RhoA-specific siRNA $(\boldsymbol{D}, \boldsymbol{E}, \boldsymbol{J}, \boldsymbol{K}, \boldsymbol{N}, \mathbf{O})$ or control, nontargeting siRNA $(\boldsymbol{B}, \boldsymbol{C}, \boldsymbol{H}, \boldsymbol{I}, \boldsymbol{L}, \boldsymbol{M})$ were transfected into axons on DIV 5 , and RhoA transcripts were detected by FISH on DIV 7.P, $\mathbf{Q}$, No retrograde transport or diffusion was observed $48 \mathrm{~h}$ after transfection of FITC-labeled control siRNA into side chamber axons. Dotted line, Border of divider. Scale bar, $10 \mu \mathrm{m}$. $\boldsymbol{R}$, Quantification of the intensity of FISH signals in side and contiguous central chamber axons transfected with RhoA-specific or control siRNA. Axons in the side compartment were transfected on 5 DIV, and FISH with a RhoA-specific probe was performed on 7 DIV. ${ }^{* *} p<0.0001$, unpaired, two-tailed $t$ test; $n \geq 27$ per condition. S, Transfection of RhoA-specific siRNA into the side chamber prevents growth cone collapse in response to Sema3A. Axons in the side compartment were transfected as above, and growth cone collapse assays were performed on $7 \mathrm{DIV}$. Sema $3 \mathrm{~A}$ $(450 \mathrm{ng} / \mathrm{ml})$ induces collapse in $60 \mathrm{~min}$ in axons transfected with control siRNA but elicits substantially reduced collapse in axons transfected with RhoA-specific siRNA. ${ }^{* *} p<0.0001$, unpaired, two-tailed $t$ test; $n=5$ optical fields with a total of $\geq 65$ growth cones per condition.

siRNA transfection (L. J. Cox, U. Hengst and Jaffrey, unpublished observations). The observed mRNA knock-down is specific because control siRNAs fail to induce mRNA knock-down. Our data do not exclude, however, the possibility that mRNA knock- down might be mediated by a novel mechanism involving siRNA-induced retrograde trafficking of axonal mRNAs.

MicroRNAs mediate translational repression by inducing the formation of RISC complexes that contain components that are 
found in siRNA-induced RISC complexes (Mello and Conte, 2004). Our finding that RNAi machinery is present in developing axons and the ability of transfected siRNA to induce functional siRNA-containing RISC complexes raises the possibility that microRNA RISC complexes might also be able to form and have roles in mRNA translation in developing axons. Our finding that Dicer is present in axons raises the possibility that premicroRNAs are processed in axons and that this processing may be a feature of intra-axonal mRNA translational regulation.

A recently recognized component of the RNAi pathway is FMRP (Jin et al., 2004a). Although the role of FMRP in RNAi is unclear, FMRP is associated with components of RISC (Jin et al., 2004a). In patients with fragile $X$ syndrome, a trinucleotide repeat expansion in the $5^{\prime} \mathrm{UTR}$ of the FMR 1 gene, which encodes FMRP, results in reduced gene transcription and near absent FMRP levels. FMRP has previously been localized to dendrites (Feng et al., 1997), in which it its absence is thought to account for the characteristic dendritic spine dysmorphogenesis in this disorder (Bagni and Greenough, 2005). Because proper connection between axons and dendrites is required for synapse and spine formation, it is interesting to speculate that defective function of axonal FMRP may contribute to the histopathologic and other neurodevelopmental abnormalities in this syndrome (Bagni and Greenough, 2005).

The RNAi proteins that we examined were localized to punctate structures reminiscent of RNA granules, neuronal structures enriched in ribosomes, mRNA, and translational machinery (Krichevsky and Kosik, 2001; Kanai et al., 2004). Because many components of RNAi have been found to be associated with ribosomes (Jin et al., 2004b; Kim et al., 2004), their punctate localization in axons may reflect their incorporation in subsets of RNA granules. Although FMRP and Dicer do not extensively colocalize with argonautes, this may reflect distinct roles for Dicer- or FMRP-containing granules. Additional studies will be required to distinguish the function of the different classes of RNA granules and to distinguish them from previously described granules, such as Staufen-containing transport granules (Kanai et al., 2004).

The presence of functional RNAi proteins in axons raises the question of the physiological role of these proteins during neuronal development. In zebrafish deficient in Dicer function, severe abnormalities in brain development are seen including defects in axonal trajectories (Giraldez et al., 2005). Although the zebrafish study did not address the subcellular localization, i.e., axonal or somatic, of the microRNA targets whose misregulation resulted in the brain morphogenesis defects, our studies raise the possibility that defective mRNA translation within axons may contribute to this phenotype. Indeed, morphologic responses of growth cones elicited by Sema3A require the intra-axonal translation of RhoA (Wu et al., 2005), a transcript that contains an unusually high number and density of miRNA binding sites (Wu et al., 2005). Conceivably, Sema3Adependent regulation of RhoA mRNA translation is regulated by RISC. Interestingly, RhoA mRNA also contains a conserved FMRPbinding domain (Chen et al., 2003).

Axonal mRNA translation has been implicated in several processes, including turning responses elicited by guidance cues (Campbell and Holt, 2001), orientation and adaptation to gradients of guidance cues (Ming et al., 2002), and BDNF-induced potentiation of neurotransmitter release (Zhang and Poo, 2002). In addition to RhoA, several other axonal mRNAs have recently been identified and proposed to have roles in axonal guidance and synaptogenesis (Taylor et al., 2005; Lyles et al., 2006; Piper et al., 2006). However, the ability to test whether specific mRNAs are required for putative biological functions is particularly dif- ficult. Our axon-specific mRNA knock-down experiments constitute a simple and straightforward method to assess the roles of specific axonal mRNAs. Although the compartmentalized Campenot system that was used in our study has been used primarily for the culture of DRG and sympathetic neurons, newer microfluidic compartmentalized systems that permit fluidic isolation of axons and cell bodies for a wide variety of neurons (Taylor et al., 2005), are likely to be useful for axon-specific mRNA knock-down experiments in other classes of neurons.

\section{References}

Antar LN, Dictenberg JB, Plociniak M, Afroz R, Bassell GJ (2005) Localization of FMRP-associated mRNA granules and requirement of microtubules for activity-dependent trafficking in hippocampal neurons. Genes Brain Behav 4:350-359.

Bagni C, Greenough WT (2005) From mRNP trafficking to spine dysmorphogenesis: the roots of fragile X syndrome. Nat Rev Neurosci 6:376-387.

Bassell GJ, Zhang H, Byrd AL, Femino AM, Singer RH, Taneja KL, Lifshitz LM, Herman IM, Kosik KS (1998) Sorting of $\beta$-actin mRNA and protein to neurites and growth cones in culture. J Neurosci 18:251-265.

Brittis PA, Lu Q, Flanagan JG (2002) Axonal protein synthesis provides a mechanism for localized regulation at an intermediate target. Cell 110:223-235.

Campbell DS, Holt CE (2001) Chemotropic responses of retinal growth cones mediated by rapid local protein synthesis and degradation. Neuron 32:1013-1026.

Campenot RB (1977) Local control of neurite development by nerve growth factor. Proc Natl Acad Sci USA 74:4516-4519.

Caudy AA, Myers M, Hannon GJ, Hammond SM (2002) Fragile X-related protein and VIG associate with the RNA interference machinery. Genes Dev 16:2491-2496.

Chen L, Yun SW, Seto J, Liu W, Toth M (2003) The fragile X mental retardation protein binds and regulates a novel class of mRNAs containing U-rich target sequences. Neuroscience 120:1005-1017.

Eng H, Lund K, Campenot RB (1999) Synthesis of $\beta$-tubulin, actin, and other proteins in axons of sympathetic neurons in compartmented cultures. J Neurosci 19:1-9.

Feng Y, Gutekunst CA, Eberhart DE, Yi H, Warren ST, Hersch SM (1997) Fragile $\mathrm{X}$ mental retardation protein: nucleocytoplasmic shuttling and association with somatodendritic ribosomes. J Neurosci 17:1539-1547.

Giraldez AJ, Cinalli RM, Glasner ME, Enright AJ, Thomson JM, Baskerville S, Hammond SM, Bartel DP, Schier AF (2005) MicroRNAs regulate brain morphogenesis in zebrafish. Science 308:833-838.

Irvin-Wilson CV, Chaudhuri G (2005) Alternative initiation and splicing in dicer gene expression in human breast cells. Breast Cancer Res 7:R563-R569.

Ishizuka A, Siomi MC, Siomi H (2002) A Drosophila fragile X protein interacts with components of RNAi and ribosomal proteins. Genes Dev 16:2497-2508.

Jin P, Alisch RS, Warren ST (2004a) RNA and microRNAs in fragile X mental retardation. Nat Cell Biol 6:1048-1053.

Jin P, Zarnescu DC, Ceman S, Nakamoto M, Mowrey J, Jongens TA, Nelson DL, Moses K, Warren ST (2004b) Biochemical and genetic interaction between the fragile $\mathrm{X}$ mental retardation protein and the microRNA pathway. Nat Neurosci 7:113-117.

Kanai Y, Dohmae N, Hirokawa N (2004) Kinesin transports RNA: isolation and characterization of an RNA-transporting granule. Neuron 43:513-525.

Kim J, Krichevsky A, Grad Y, Hayes GD, Kosik KS, Church GM, Ruvkun G (2004) Identification of many microRNAs that copurify with polyribosomes in mammalian neurons. Proc Natl Acad Sci USA 101:360-365.

Kleiman R, Banker G, Steward O (1994) Development of subcellular mRNA compartmentation in hippocampal neurons in culture. J Neurosci 14:1130-1140.

Krichevsky AM, Kosik KS (2001) Neuronal RNA granules: a link between RNA localization and stimulation-dependent translation. Neuron 32:683-696.

Krichevsky AM, King KS, Donahue CP, Khrapko K, Kosik KS (2003) A 
microRNA array reveals extensive regulation of microRNAs during brain development. RNA 9:1274-1281.

Lyles V, Zhao Y, Martin KC (2006) Synapse formation and mRNA localization in cultured Aplysia neurons. Neuron 49:349-356.

Mello CC, Conte Jr D (2004) Revealing the world of RNA interference. Nature 431:338-342.

Ming GL, Wong ST, Henley J, Yuan XB, Song HJ, Spitzer NC, Poo MM (2002) Adaptation in the chemotactic guidance of nerve growth cones. Nature 417:411-418.

Olink-Coux M, Hollenbeck PJ (1996) Localization and active transport of mRNA in axons of sympathetic neurons in culture. J Neurosci 16:1346-1358.

Piper M, Anderson R, Dwivedy A, Weinl C, van Horck F, Leung KM, Cogill E, Holt C (2006) Signaling mechanisms underlying Slit2-induced collapse of Xenopus retinal growth cones. Neuron 49:215-228.

Steward O (2002) Translating axon guidance cues. Cell 110:537-540.
Tanaka Hall TM (2005) Structure and function of argonaute proteins. Structure 13:1403-1408.

Taylor AM, Blurton-Jones M, Rhee SW, Cribbs DH, Cotman CW, Jeon NL (2005) A microfluidic culture platform for CNS axonal injury, regeneration and transport. Nat Methods 2:599-605.

Weiler IJ, Irwin SA, Klintsova AY, Spencer CM, Brazelton AD, Miyashiro K, Comery TA, Patel B, Eberwine J, Greenough WT (1997) Fragile X mental retardation protein is translated near synapses in response to neurotransmitter activation. Proc Natl Acad Sci USA 94:5395-5400.

Wu K, Hengst U, Cox LJ, Macosko EZ, Jeromin A, Urquhart ER, Jaffrey SR (2005) Local translation of RhoA regulates growth cone collapse. Nature 436:1020-1024.

Yamada KM, Spooner BS, Wessells NK (1971) Ultrastructure and function of growth cones and axons of cultured nerve cells. J Cell Biol 49:614-635.

Zhang X, Poo MM (2002) Localized synaptic potentiation by BDNF requires local protein synthesis in the developing axon. Neuron 36:675-688. 